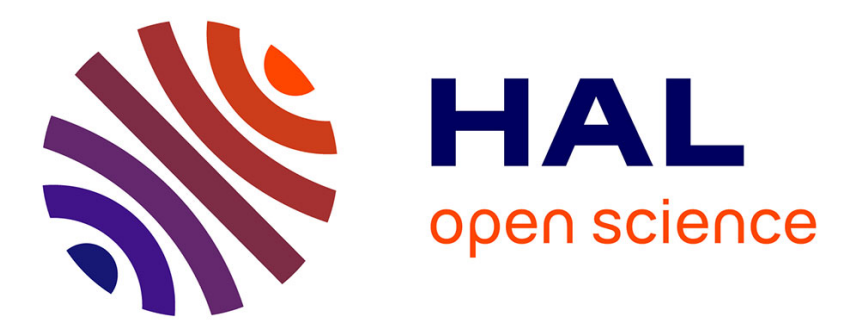

\title{
Effects of stretching velocity on passive resistance developed by the knee musculo-articular complex: contributions of frictional and viscoelastic behaviours
}

\author{
Antoine Nordez, Pascal Casari, Christophe Cornu
}

\section{- To cite this version:}

Antoine Nordez, Pascal Casari, Christophe Cornu. Effects of stretching velocity on passive resistance developed by the knee musculo-articular complex: contributions of frictional and viscoelastic behaviours. European Journal of Applied Physiology, 2008, 103 (2), pp.243-250. 10.1007/s00421008-0695-9 . hal-01006781

\section{HAL Id: hal-01006781 \\ https://hal.science/hal-01006781}

Submitted on 16 Jun 2014

HAL is a multi-disciplinary open access archive for the deposit and dissemination of scientific research documents, whether they are published or not. The documents may come from teaching and research institutions in France or abroad, or from public or private research centers.
L'archive ouverte pluridisciplinaire HAL, est destinée au dépôt et à la diffusion de documents scientifiques de niveau recherche, publiés ou non, émanant des établissements d'enseignement et de recherche français ou étrangers, des laboratoires publics ou privés.

\section{(c)(1)}

Distributed under a Creative Commons Attribution| 4.0 International License 


\title{
Effects of stretching velocity on passive resistance developed by the knee musculo-articular complex: contributions of frictional and viscoelastic behaviours
}

\author{
A. Nordez $\cdot$ P. Casari $\cdot$ C. Cornu
}

\begin{abstract}
It is commonly accepted that the passive musculo-articular complex (MAC) displays a viscoelastic behavior. However, the viscosity of the MAC is still not well understood when considering the relationship between the passive resistance offered by the MAC and the stretching velocity. Therefore, in order to obtain a better knowledge of the mechanical behavior of the passive MAC, nine subjects performed passive knee extension/flexion cycles with the hip angle set at $60^{\circ}$ on a Biodex ${ }^{\circledR}$ dynamometer at $5^{\circ}, 30^{\circ}, 60^{\circ}, 90^{\circ}$ and $120^{\circ} \mathrm{s}^{-1}$ in a randomized order to $80 \%$ of their maximal range of motion. Results show significant $(P<0.001)$ increases with the stretching velocity for the passive torque (between +17.6 and $+20.8 \%$ depending on the considered knee angle), the potential elastic energy stored during the loading (E: $+22.7 \%)$, and the dissipation coefficient (DC: $+22.8 \%$ ). These results suggest that the role of viscosity in the MAC's mechanical behavior is limited. A linear model was well-fitted on torque-velocity $\left(0.93<R^{2}<0.98\right)$, E-velocity $\left(R^{2}=0.93\right)$ and DC-velocity $\left(R^{2}=0.99\right)$ relationships. The linear relationship between DC and velocity indicates that the DC does not tend towards zero for the slowest velocities and that the dissipative properties of the MAC could be modeled
\end{abstract}

A. Nordez $\cdot$ C. Cornu

Université de Nantes, Nantes Atlantique Universités,

Laboratoire "Motricité, Interactions, Performance", JE 2438,

UFR STAPS, 25 bis Bd Guy Mollet BP 72206,

44000 Nantes, France

e-mail: christophe.cornu@univ-nantes.fr

P. Casari

Université de Nantes, Nantes Atlantique Universités, CNRS, Institut de recherche en Génie Civil et Mécanique, UMR 6183, UFR des Sciences et Techniques,

2 rue de la Houssinière BP 92208, 44000 Nantes, France by combining linear viscosity and friction. The present study would allow the implementation of a rheological model to simulate the behavior of the passive MAC.

Keywords Passive torque - Dissipative properties . Viscosity $\cdot$ Friction $\cdot$ Elasticity

\section{Introduction}

In human biomechanics, the passive mechanical properties of a musculo-articular complex (MAC), including structures spanning the joint (Riemann et al. 2001), can be determined by using the relationship between the articular angle and the passive torque developed by the MAC in resistance to motion (Gajdosik 2001; Gajdosik et al. 2004, 2005; Magnusson 1998; Magnusson et al. 1995, 1996a, 1998, 2000a, b; McNair et al. 2001, 2002; McNair and Portero 2005; Nordez et al. 2006, 2008b). These mechanical properties have been frequently discussed in literature, for instance, to study changes in flexibility induced by ageing (Gajdosik et al. 2004, 2005), spasticity and trauma (Bressel et al. 2004; Bressel and McNair 2001; Rabita et al. 2005), or acute (Magnusson et al. 1995, 1996a, 2000b; McNair et al. 2001, 2002; Nordez et al. 2006, 2008b) and chronic (Gajdosik et al. 2004; Magnusson et al. 1996b; Reid and McNair 2004) stretching protocols.

Considering the torque relaxation during a static stretching protocol (Gajdosik et al. 2004; Magnusson 1996a, 1998, 2000a; McNair et al. 2001) and the dissipation of energy during a loading/unloading cycle (Magnusson et al. 1995, 1998; Nordez et al. 2008b), results of many studies have indicated that the MAC displays a viscoelastic behavior. A viscoelastic material displays both relaxation during static stretching and dissipation during a 
loading/unloading cycle. Nevertheless, some other mechanical behaviors can theoretically partially explain these phenomena such as plasticity and friction. Since plasticity involves irreversible alterations, it can be proposed that both the isolated muscle-tendon unit (Taylor et al. 1990) and the in vivo musculo-articular complex (Esteki and Mansour 1996; Gajdosik 2001; Magnusson et al. 1998; McNair et al. 2002; Nordez et al. 2008b) do not exhibit plastic behavior when muscles are stretched in physiological conditions. However, the dissipation of energy during a loading/unloading cycle could also partially be due to friction (Esteki and Mansour 1996).

Figure 1 shows the effects of elasticity, viscosity and friction on the relationships between angular velocity and the potential elastic energy stored during the loading (E, i.e., the area under the loading torque-angle curve), and between angular velocity and the dissipation coefficient (DC) calculated as the energy dissipated (i.e., hysteresis area) normalized by $E$ (Mandel 1969; Persoz 1960). The mechanical behaviour of the MAC could be considered in a number of ways. (1) A purely elastic behavior means that $E$ does not depend on velocity and that there is no energy dissipation. (2) A viscoelastic behavior with a linear viscosity implies that both $E$ and DC increase linearly with velocity, without any dissipation for very low velocities. (3) A viscoelastic behavior with a non linear viscosity would be similar to (2) but with non linear increases in $E$ and DC with velocity. (4) A viscoelastic behavior (linear viscosity) with friction would imply linear relationships between $E$, DC and the velocity with y-intercepts higher than zero. Consequently, the study of changes in $E$ and DC with stretching velocity should provide fundamental information about the mechanical behavior of the studied structures.

As stated above, the viscoelasticity of the MAC would imply that the passive torque (or E) is increased with stretching velocity (Fig. 1). However, the effect of stretching velocity on the passive torque has been little

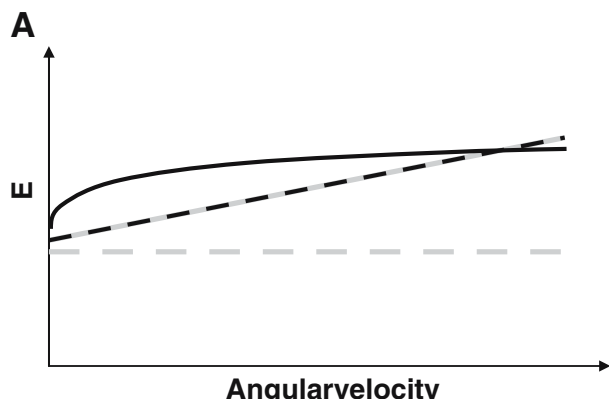

Fig. 1 Different mechanical behavior to model the dissipation of the musculo-articular complex during a loading/unloading cycle. a Potential elastic energy stored during the loading $(E)$ and, b dissipation coefficient $(D C)$ as function of the angular velocity. studied. In fact, only one study concerning the knee joint has shown that stiffness during passive extension (i.e., the slope of the torque-angle curve) was not different when considering two stretching velocities $\left(5^{\circ}\right.$ and $20^{\circ} \mathrm{s}^{-1}$ ) (Magnusson et al. 1998). On the contrary, two studies have shown that both passive torque (McNair et al. 2002; Singer et al. 2003) and stiffness (McNair et al. 2002) were increased by about 12 and $16 \%$, respectively between $5^{\circ}$ and $25^{\circ} \mathrm{s}^{-1}$ during passive ankle dorsi-flexion for middleaged men and women. Moreover, another study has shown an increase in passive torque during the dorsi-flexion of about $17.5 \%$ between $5^{\circ}$ and $120^{\circ} \mathrm{s}^{-1}$ for young women (Gajdosik et al. 2005). Nevertheless, sparse data are available regarding the relationship between passive torque and stretching velocity, in a given range of velocities. To our knowledge, only two studies have established this relationship in order to assess the effect of velocity on the passive resistive torque developed by spastic plantar flexors (Lamontagne et al. 1997; Rabita et al. 2005). For unknown reasons, these two studies performed on similar populations (i.e., spastic and healthy middle-aged men and women) provided quite different results. For healthy subjects, Lamontagne et al. (1997) have shown that the passive torque increases linearly with velocity $(r=0.92)$ of about $700 \%$ between $10^{\circ}$ and $180^{\circ} \mathrm{s}^{-1}$. On the other hand, the increase reported by Rabita et al. (2005) was not significant for velocities lower than $161^{\circ} \mathrm{s}^{-1}$ and a relative increase of about $16 \%$ was found between $10^{\circ}$ and $211^{\circ} \mathrm{s}^{-1}$. It must be noticed that, in these two studies, torque measurements were performed for ankle angles below $0^{\circ}$ in dorsi-flexion $\left(0^{\circ}\right.$ : foot perpendicular to the leg) so that data were obtained for joint positions which would place tissues under limited strain (McNair et al. 2002). It may be then hypothesized that changes in passive torque with stretching velocity might have been angle dependent. Therefore, the torque-velocity-angle relationship should be characterized to assess the potential angle dependence relative to the effects of stretching velocity.

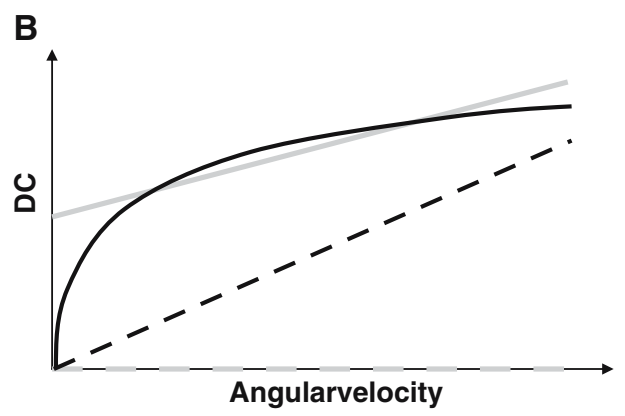

Gray dotted line purely elastic behavior, black dotted line viscoelastic behavior (linear viscosity), black line viscoelastic behavior (non linear viscosity), gray line viscoelastic behavior (linear viscosity) and friction 
As it is shown on Fig. 1, the full characterization of passive MAC mechanical behavior requires the determination of the velocity dependence of the energy dissipated by the MAC during a loading/unloading cycle. To our knowledge, only one study has determined such a relationship for the metacarpophalangeal joint. Esteki and Mansour (Esteki and Mansour 1996) have shown that the hysteresis is velocity independent at high stretching velocities $\left(50^{\circ}-75^{\circ} \mathrm{s}^{-1}\right)$ indicating that the dissipation of the potential elastic energy is frictional when studies are conducted in this range of velocities, while the dissipation of energy is linearly related to the velocity at a low angular velocity range $\left(3^{\circ}-50^{\circ} \mathrm{s}^{-1}\right)$ (Esteki and Mansour 1996). Therefore, while the mechanical behavior of the passive MAC is classically assumed to be viscoelastic in the literature (Gajdosik et al. 2005; Magnusson 1998, 2000a; McNair et al. 2001, 2002), the possible contribution of friction in this behavior has to be determined.

The objective of the present study was to assess the effect of stretching velocity on passive torque, $E$ and DC for the knee joint. Results of this study would provide a fundamental understanding of the mechanical properties of the passive MAC and particularly concerning the possible contributions of both viscoelastic and friction behaviors.

\section{Materials and methods}

\section{Subjects}

Ten healthy males $(25.4 \pm 3.0$ years, height: $182.4 \pm$ $7.5 \mathrm{~cm}$, weight: $76.6 \pm 8.1 \mathrm{~kg}$ ) volunteered to participate in this study and signed an informed consent form. This study was conducted according to the Helsinki Statement (1964). Subjects practiced recreational sports, but did not participate in any strength or flexibility training at the time of the study. No subject had sustained a recent injury that may have affected the findings.

\section{Measurement techniques}

The experimental set-up has been previously described (Nordez et al. 2006, 2008b) and has been adapted from previous studies (Magnusson et al. 1996a, 1998; Reid and McNair 2004). Briefly, the Biodex system 3 research $^{\circledR}$ isokinetic dynamometer (Biodex medical, Shirley, N.Y., USA) was used to measure torque produced in resistance to passive stretch $(\mathrm{T})$, knee joint angle $(\theta)$ and knee joint angular velocity $(\omega)$. Subjects were seated and the thigh was fastened using velcro straps to a thigh pad elevating it from horizontal. The trunk-thigh angle was adjusted to $60^{\circ}$, and the input axis of the dynamometer was aligned with the approximate axis of rotation of the knee joint. All subjects were unable to reach full knee extension in this position. All procedures began with the lower leg perpendicular to the thigh. This position was used to determine our reference knee angle expressed as $0^{\circ}$.

Surface electromyographic signals (sEMG) of hamstring muscles were also recorded synchronously with the torque and angle data to ensure that no undesirable activation occurred during the stretching protocol. Bipolar electrodes (Delsys DE 02.3, Delsys Inc, Boston, USA) were placed on semitendinosus and biceps femoris muscles with a $10 \mathrm{~mm}$ inter-electrode distance according to the recommendations of the Surface Electromyography for the Non-Invasive Assessment of Muscles project (SENIAM) (Hermens et al. 2000). In order to normalize the sEMG data recorded during the passive stretching trials, sEMG data were also collected during three maximal isometric knee flexions performed with the leg perpendicular to the thigh. T, $\theta, \omega$ and sEMG signals were sampled at $1,000 \mathrm{~Hz}$ with an Analog/Digital converter (Bagnoli 16, Delsys Inc, Boston, USA). All of the collected data were stored in a computer hard drive for further analysis; sEMG signals were also visualized in real time by the researcher and the subject.

\section{Experimental protocol}

The experimental protocol is described in Fig. 2. The maximal range of motion (ROM) was first determined (Nordez et al. 2006). The lower leg was passively extended $\left(\omega=5^{\circ} \mathrm{s}^{-1}\right)$, and the subjects used a stop switch when they perceived the maximum tolerable hamstring muscle stretch. This point was operationally defined as the maximal passive knee extension. The leg was then immediately returned to the starting position. The best score of three trials was considered as the maximal ROM. Subjects then performed a familiarization with the stretching protocol at the different tested velocities. Using the sEMG feedback, the subjects visualized sEMG activity of the muscles, and they were asked to stay as relaxed as possible. A $10 \mathrm{~min}$ rest period was then respected between the familiarization and the measurements. Five passive loading/unloading cycles were performed at $5^{\circ}, 30^{\circ}, 60^{\circ}, 90^{\circ}$ and $120^{\circ} \mathrm{s}^{-1}$ in a randomized order until $80 \%$ of the maximal ROM (Gajdosik et al. 2005). Since a pilot experiment has shown that a 5 min rest was sufficient to counteract the effects of five cyclic stretching repetitions, 5 min rests were observed between each series at a given angular velocity. To check that no changes in the passive mechanical properties occurred during the protocol, the subjects then performed five cycles at the same angular velocity as the first tested velocity. Finally, for sEMG normalization purpose, the subjects performed three maximal isometric knee flexions with the leg perpendicular to the shank and a one min rest in between each. 


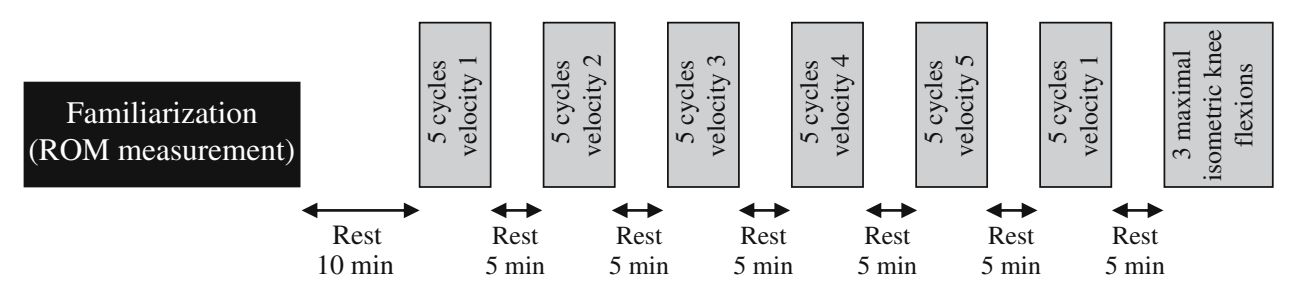

Fig. 2 Experimental protocol. The maximal range of motion (ROM) was first determined. Subjects realized then a familiarization with the stretching protocol. A 10 min rest period was then performed before starting the actual measurements. Five passive loading/unloading cycles were performed at $5^{\circ}, 30^{\circ}, 60^{\circ}, 90^{\circ}$ and $120^{\circ} \mathrm{s}^{-1}$ in a randomized order until $80 \%$ of the maximal $R O M$. Then, subjects

\section{Data analysis}

The first cycle was analyzed for all of the velocities. Data were processed and calculated using a standardized software with Matlab (The Mathworks, Natick, USA). Mechanical signals (T, $\theta$ and $\omega$ ) were filtered using a Butterworth second order low-pass-dual filter $(10 \mathrm{~Hz})$. Recorded torque was then corrected from the limb mass and inertia. For that purpose, the torque due to the limb mass was estimated in the starting position (i.e., trunk-thigh angle: $60^{\circ}$, leg-thigh angle: $90^{\circ}$, the leg $30^{\circ}$ above horizontal) at the end of the protocol. In this position, it was assumed that there is negligible hamstring and quadriceps tension. Based on this limb mass estimation, the passive torque was corrected using the cosine function of the angle between the thigh and horizontal plane (Aagaard et al. 1995). The inertia of the Biodex level arm was determined during cycles performed at different velocities during an experiment with no subject on the dynamometer. The moment of inertia of the leg-foot was determined using regression equations (McFaull and Lamontagne 1998) provided by Zatsiorski (1998).

A typical torque-angle relationship corrected from gravity and inertia is shown in Fig. 3. This figure also performed 5 cycles at the same angular velocity than the first tested velocity. A 5 min rest period was respected between each tested stretching velocity. Finally, subjects performed three maximal isometric knee flexions in order to normalize surface electromyographic signals

shows acceleration and deceleration phases. At high velocities, a deceleration artifact is present, and a previous study (Nordez et al. 2008a) has shown, using the same methodology as in the present study, that the deceleration phase was lower than $9^{\circ}$ at $120^{\circ} \mathrm{s}^{-1}$ (Fig. 3). Therefore, the initial and final $9^{\circ}$ were excluded from the range of motion used for all data analyses in order to avoid taking the deceleration artifact into account. Then, for each preset velocity, the passive torque was determined every $2^{\circ}$ within the considered joint amplitude in order to obtain the three dimensional relationship between passive torque, angle and the preset velocity. The average of the recorded angular velocity during the loading was also calculated as a function of the preset velocity. For all velocities, the energy stored during loading ( $E$, i.e., the area under the loading curve), the energy restituted (ER, i.e., the area under the unloading curve) and the energy dissipated by the musculoarticular complex (ED, i.e., the hysteresis area) were calculated. ED was divided by $E$ to calculate the dissipation coefficient [DC, Eq. (1), (Nordez et al. 2008b)].

$\mathrm{DC}=\mathrm{ED} / E=(E-\mathrm{ER}) / E$

The inertia of the leg was estimated using predictive equations (Zatsiorski 1998). Since the acceleration phase is

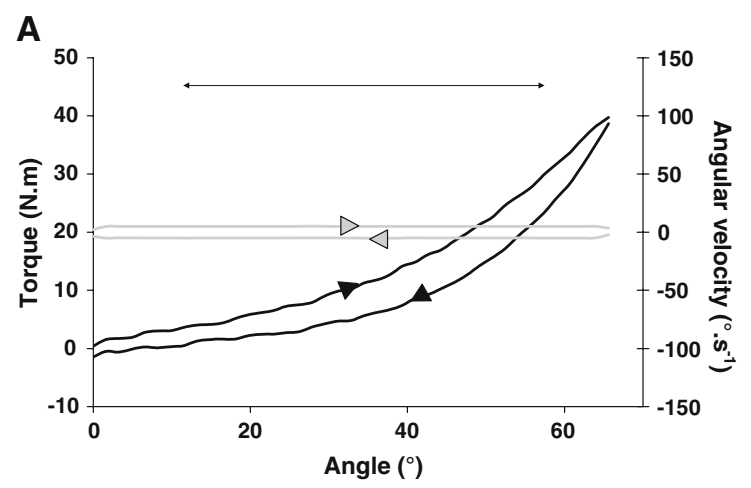

Fig. 3 Typical examples of a torque-angle relationships corrected for gravity and inertia (black line), and of a velocity patterns (gray line) performed for preset angular velocities of $5^{\circ} \mathrm{s}^{-1}$ (a) and $120^{\circ} \mathrm{s}^{-1}$ (b).

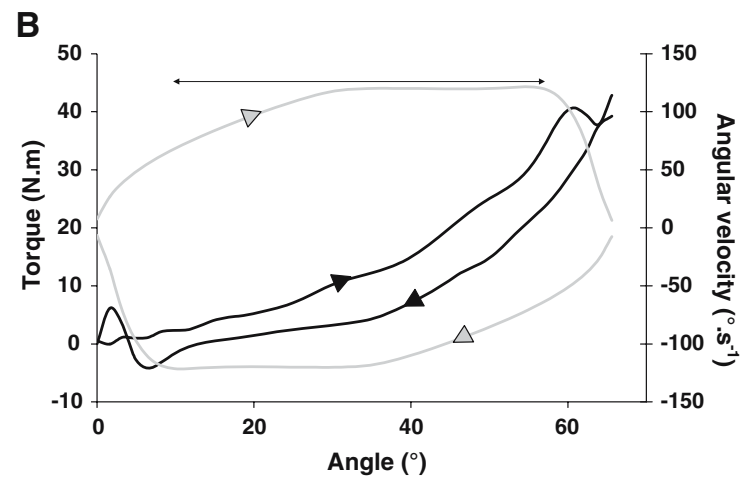

Right left arrow range of motion used for all data analyses to avoid taking into account deceleration artifacts 
taken into account for the calculation of $E$ and DC (Fig. 3), the estimation of the inertia might have significantly influenced the findings. Therefore, a sensitivity analysis was conducted and we calculated $E$ and DC values for an inertia overestimated and under estimated of approximately $20 \%$. The $20 \%$ calculated is higher than the difference classically reported in the literature between personalized inertia measurement using imaging, and inertia estimated using predictive equations, e.g., (Dumas et al. 2005).

sEMG data were processed according to the procedure described by Gajdosik et al. (Gajdosik et al. 2005). The root mean square of sEMG signals (sEMG RMS), calculated during stretching, was deduced from the sEMG RMS calculated during a 2-s window while the subject was totally relaxed. sEMG RMS values were then normalized with the maximal level reached during the maximal isometric contractions. If sEMG RMS for a subject was greater than $1 \%$ of the maximum voluntary contraction during stretching trials, then the subject's data were discarded (McNair et al. 2001, 2002).

\section{Statistical analysis}

After checking the distribution of data (KolmogorovSmirnov test), parametric statistical tests were performed. In order to check that the MAC's mechanical properties were not altered during the protocol, $E$ and DC calculated for the first and the last series (same velocity for a subject) were compared using a paired $t$-test. The effect of velocity on the passive torque for three knee angles $\left(28^{\circ}, 40^{\circ}\right.$ and $52^{\circ}$; these three knee angles were within the isokinetic phase during the loading for all tested velocities), $E$ and DC were determined using five ANOVAs with repeated measures on velocity. Newman-Keuls post-hoc analyses were performed when appropriate. The critical level of significance in the present study was set at $P<0.05$.

\section{Results}

Table 1 shows averaged velocities as function of the preset velocities on the ROM considered for the data analysis. Thus, at the maximal preset velocity used in the present study $\left(120^{\circ} \mathrm{s}^{-1}\right)$, the mean measured velocity was about $106.6^{\circ} \mathrm{s}^{-1}$. These averaged velocities were used to establish the relationships between $E$, DC and the velocity.
Two subjects were excluded from the study because their sEMG RMS levels during the passive stretching protocol were higher than $1 \%$ of the maximal level reached during isometric contractions. No significant difference was found between the first and the last series of cycles performed at the same angular velocity for $E(P=0.326$; $9.08 \pm 2.62 \mathrm{~J}$ vs. $9.37 \pm 2.58 \mathrm{~J})$ and $\mathrm{DC}(P=0.214$; $0.57 \pm 0.07$ vs. $0.53 \pm 0.09$ ).

At a preset velocity of $120^{\circ} \mathrm{s}^{-1}$, the mean normalized differences between $E, E$ estimated with inertia overestimated at $20 \%$, and with inertia underestimated at $20 \%$ were $-1.2 \pm 0.4 \%$ and $+1.5 \pm 0.4 \%$, respectively. For DC, those differences were $-3.8 \pm 1.6 \%$ and $3.4 \pm 1.2 \%$, respectively.

The mean torque-angle-velocity is depicted in Fig. 4a. A significant main effect of velocity $(P<0.001)$ was found for the passive torque at the three considered angles indicating that the passive torque is increased with stretching velocity (Fig. 4 b). Increases in passive torque between $5^{\circ}$ and $120^{\circ} \mathrm{s}^{-1}$ were $+17.6 \pm 7.1 \% \quad\left(28^{\circ}\right.$ knee angle $)$, $+17.8 \pm 6.3 \%\left(40^{\circ}\right.$ knee angle $)$ and $+20.8 \pm 3.1 \%\left(52^{\circ}\right.$ knee angle). For these three knee angles, a linear relationship was observed for the mean torque-velocity relationship $\left(0.93<\mathrm{R}^{2}<0.98, P<0.01\right)$.

$E$ and DC were also increased $(P<0.001)$ with the stretching velocity $(+22.7 \pm 5.5 \%$ and $+22.8 \pm 14.2 \%$ between $5^{\circ}$ and $120^{\circ} \mathrm{s}^{-1}$ for $E$ and DC, respectively). Linear relationships were observed for the averaged $E$ velocity (Fig. 5a) and DC-velocity (Fig. 5b) relationships $\left(R^{2}=0.93, \quad P<0.01\right.$ and $R^{2}=0.99, \quad P<0.001$, respectively).

\section{Discussion}

The present study shows that the passive torque developed in resistance to the stretch, $E$ and the DC, are increased with stretching velocity. Since it has been shown that repeated cyclic stretching induce changes in MAC mechanical properties (Magnusson et al. 1998; McNair et al. 2001, 2002; Nordez et al. 2008b), it could be argued that some cyclic stretching effects would interfere with the effects of stretching velocity. Our results show that there were no significant changes between the first and the last series of cycles. Consequently, in accordance with our pilot experiment, it can be considered that the $5 \mathrm{~min}$ rest period

Table 1 Averaged angular velocity in the range of motion of interest for data analysis (see text) as function of the preset velocity

\begin{tabular}{llllll}
\hline Preset angular velocity $\left({ }^{\circ} \mathrm{s}^{-1}\right)$ & 5 & 30 & 60 & 90 & 120 \\
Averaged measured angular velocity $\left({ }^{\circ} \mathrm{s}^{-1}\right)$ & $5.0 \pm 0.0$ & $29.4 \pm 0.1$ & $59.0 \pm 0.1$ & $85.0 \pm 0.7$ & $106.6 \pm 1.1$ \\
\hline
\end{tabular}

Velocity is presented as the mean ( \pm standard deviation) for all the subjects 


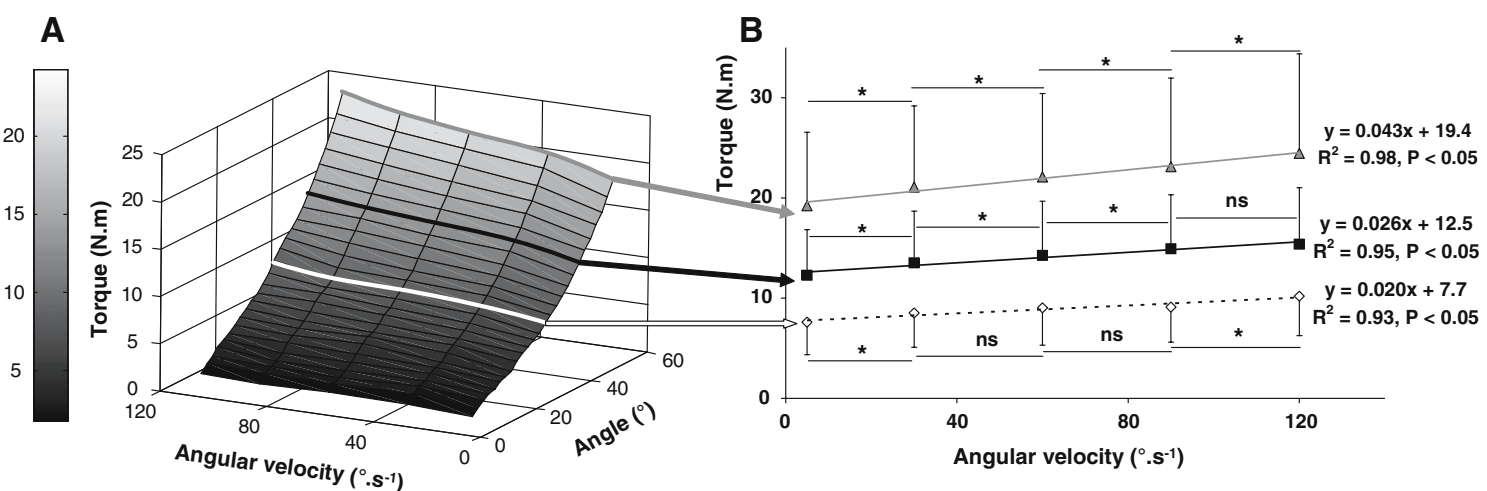

Fig. 4 a Averaged torque-angle-angular velocity relationship. b Mean torque-angular velocity relationships determined at $28^{\circ}$ (diamond), $40^{\circ}$ (square) and $52^{\circ}$ (triangle) knee angle. $* P<0.05$, ns: $P>0.05$

between each series was sufficient to counteract the changes induced by the five loading/unloading cycles. In addition, $E$ and DC were changed very little when the inertia was overestimated and underestimated by $20 \%$. It could be then concluded that inertia estimation using predictive equations, and acceleration phase had negligible influence on our results.

Increases in passive torque with stretching velocity (between +17.6 and $+20.8 \%$ ) and $E(+22.7 \%)$ reported in the present study are in the same range as those reported by Gajdosik et al. (Gajdosik et al. 2005) during passive dorsiflexion in healthy young women (passive torque: $+17.5 \%$; $\mathrm{E}:+13 \%)$. Our results are also in agreement with those of Lamontagne et al. (1997), showing that during passive dorsi-flexion for healthy middle aged subjects, the increase in passive torque with the velocity could be modeled using a linear relationship. However, in this latter study, the slope of the passive torque-velocity relationship was generally higher (an increase of about $700 \%$ between $10^{\circ}$ and $180^{\circ} \mathrm{s}^{-1}$ at $0^{\circ}$ of ankle angle during a passive dorsi-flexion) than in our present study (between $+17.6 \%$ and $+20.8 \%$ depending on the considered knee angle). The discrepancies between these results remain unexplained since the mean torque-angle-velocity relationship (Fig. 3a) did not show that the increase in passive torque with the angular velocity was angle dependent.

The increases in passive torque and $E$ with velocity confirm that the MAC displays a viscoelastic behavior (McNair et al. 2002). However, increases in passive torque, $E$ and DC, are only approximately $20 \%$, while the stretching angular velocity was multiplied by more than 20 . These results suggest that the passive torque has relatively little velocity dependence, and that viscosity does not play a major role in the passive mechanical behavior of the MAC.

In order to investigate the relative contribution of viscosity in the energy dissipation of the MAC, we determined the DC-velocity relationship (Fig. 1). The data in Fig. 5b provide the evidence that a dissipation of energy is still found even for slow stretching velocities. Indeed, the linear model, which is well-fitted to the experimental relationship $\left(R^{2}=0.99\right)$, predicts an averaged DC of 0.438 for an angular velocity of zero. This result is similar to the DC value reported by two previous studies (Magnusson et al. 1998; Nordez et al. 2008b), which have determined a DC of about 0.45 at $5^{\circ} \mathrm{s}^{-1}$ using a similar methodology on
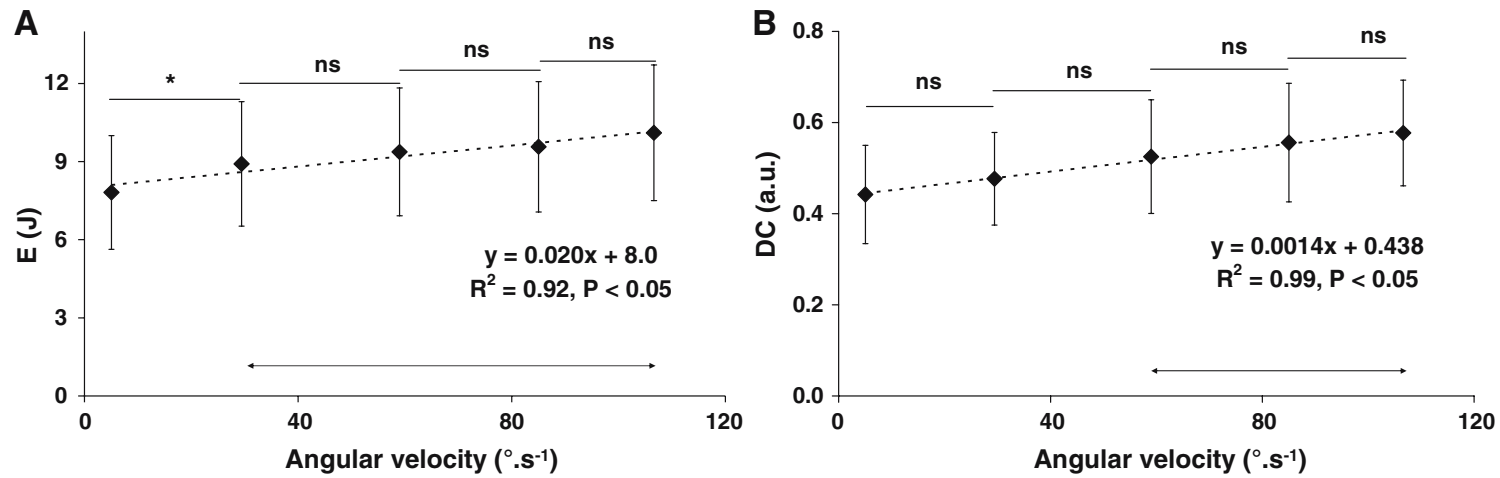

Fig. 5 a Averaged potential elastic energy stored during loading $(E)$ and $\mathbf{b}$ averaged dissipation coefficient $(D C)$ as function of stretching velocity. $* P<0.05$, ns: $P>0.05$, right left arrow range significantly $(P<0.05)$ different from the lowest tested velocity $\left(5^{\circ} \mathrm{s}^{-1}\right)$ 
similar subjects as in the present study. These results emphasize that the dissipation of energy cannot be modeled only by a linear viscosity (Fig. 1) (Mandel 1969; Persoz 1960).

Therefore, two different ways can be used to model passive MAC energy dissipation. Some authors have proposed to use a non-linear function (power model) on the DC-velocity relationship to model the dissipation-angular velocity relationship as shown in Fig. 1, illustrated by Eq. (2) (Amankwah et al. 2004; Esteki and Mansour 1996).

$\mathrm{DC}=\omega^{n}$

Where $\omega$ is the angular velocity and $n$ is a constant value.

This model was used for our experimental results (Fig. 6), but the result was that it did not give as good a fit as the linear model $\left(R^{2}=0.91\right.$ vs. $\left.R^{2}=0.99\right)$, and it predicts a DC of 0 for very slow velocities. Moreover, Esteki and Mansour (1996) have considered that a power value $(n)$ of 0 would indicate that the dissipation solely originates from a frictional behavior, and a power value of 1 would be an indication of a linear viscous dissipation. Since we find a very low power value $(0.085)$, the power model confirms that knee MAC behavior has little velocity dependence, indicating that the role of viscosity is limited, and that a significant frictional behavior has to be taken into account. Therefore, in order to model the dissipation of the MAC, a linear fit was preferentially used, indicating that the mechanical behavior of the passive MAC could be modeled by combining both a frictional and a linear viscosity behavior (Fig. 1) (Mandel 1969; Persoz 1960). Thus, the dissipation due to the friction is velocity independent (y-intercept of the DC-velocity relationship) and the dissipation modeled by the viscosity is velocity dependent (slope of the DC-velocity relationship).

Although our results indicate that a significant friction is present in MAC mechanical behavior, the relative contributions of the different structures to this friction remain

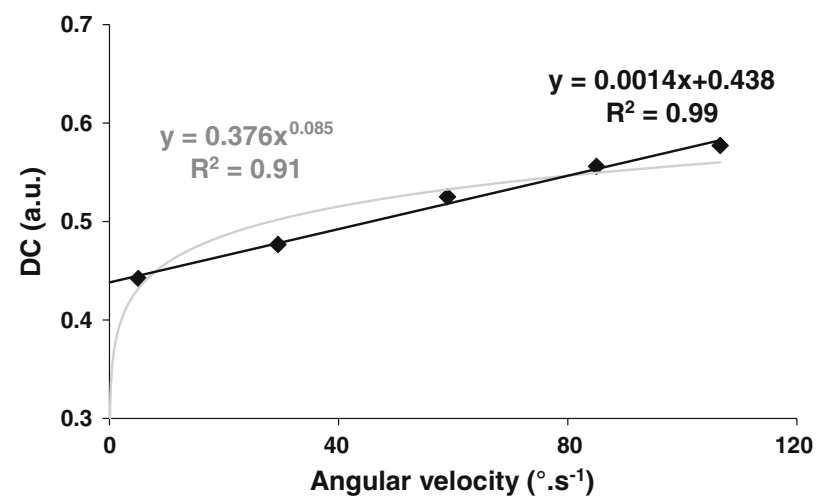

Fig. 6 Dissipation coefficient (DC) as function of the averaged angular velocity. Black line linear fit used in the present study, gray line power fit used in the study of Esteki and Mansour unknown and cannot be determined from the present study. Some friction could be due to the joint. Nevertheless, it has been shown that lubrication leads to a very low joint friction coefficient (Fung 1983). However, muscle and ligament tensions are increased during stretching, leading to an increase in articular pressure and then possibly in joint friction. In addition, during joint motion the relative displacements of the different tissues may also partially explain the friction behavior of the MAC.

It is well known that the stretch reflex is increased with the stretch velocity. For instance, Hufschmidt and Mauritz (1985) only noticed stretch reflex events for movement velocities above $80^{\circ} \mathrm{s}^{-1}$. However, Gajdosik et al. (2005) have shown that it is possible to perform stretching without any measurable sEMG activities considering the range of $5^{\circ}$ and $120^{\circ} \mathrm{s}^{-1}$ of stretching velocities. Therefore, in our study, the same angular velocity range was considered. While no significant muscle activity was observed in our study, whatever the considered stretching velocity, it could be argued that a very slight muscle activity (less than $1 \%$ of the maximal levels reached during maximal contractions), lower than the acceptable threshold commonly reported in literature (Gajdosik et al. 2005; McNair et al. 2001, 2002; McNair and Portero 2005; Nordez et al. 2008b), might have slightly influenced our results (Gajdosik 2001). Nevertheless, in this case, it would have led to a slight overestimation of the passive torque at high velocities, and then, to a slight overestimation of the relative contribution of viscosity in respect to friction. Therefore, it would not have influenced the main finding of the present study indicating that the behavior of the MAC is little dependence on velocity, and it would have induced a slight overestimation of the relative contribution of viscosity with respect to friction.

\section{Conclusion}

The results of the present study show that passive torque, $E$ and DC are significantly increased with the stretching velocity and confirm the viscoelastic behavior of the passive MAC classically accepted and reported in the literature. However, magnitudes of increase in these parameters are only about $20 \%$ when the stretching velocity is multiplied by more than 20 indicating that the contribution of viscosity in passive MAC's mechanical behavior is limited. In addition, since our original results show a linear increase in DC with the velocity, the mechanical behavior can be modeled by a viscoelasticity (with linear viscosity) combined with some friction. Future studies should be developed to determine the relative changes in viscosity and friction induced by acute (e.g., cyclic and static stretching protocols) or chronic solicitations (e.g., ageing). The present study provides 
important information for the modeling of the mechanical behavior of the MAC, and could be a first step in the implementation of a rheological model to simulate the behavior of the passive MAC.

Acknowledgments The authors thank Jacques Guilbaud for correcting the English and Michel Roche for his technical assistance.

\section{References}

Aagaard P, Simonsen EB, Trolle M, Bangsbo J, Klausen K (1995) Isokinetic hamstring/quadriceps strength ratio: influence from joint angular velocity, gravity correction and contraction mode. Acta Physiol Scand 154:421-427

Amankwah K, Triolo RJ, Kirsch R (2004) Effects of spinal cord injury on lower-limb passive joint moments revealed througth a nonlinear viscoelastic model. J Rehabil Res Dev 41:15-32

Bressel E, McNair PJ (2001) Biomechanical behavior of the plantar flexor muscle-tendon unit after an Achilles tendon rupture. Am J Sports Med 29:321-326

Bressel E, Larsen BT, McNair PJ, Cronin J (2004) Ankle joint proprioception and passive mechanical properties of the calf muscles after an Achilles tendon rupture: a comparison with matched controls. Clin Biomech (Bristol, Avon) 19:284-291

Dumas R, Aissaoui R, Mitton D, Skalli W, de Guise JA (2005) Personalized body segment parameters from biplanar low-dose radiography. IEEE Trans Biomed Eng 52:1756-1763

Esteki A, Mansour JM (1996) An experimentally based nonlinear viscoelastic model of passive joint moment. J Biomech 29:443450

Fung YC (1983) Biomechanics. Mechanical properties of living tissues. Springer, Berlin

Gajdosik RL (2001) Passive extensibility of skeletal muscle: review of the literature with clinical implications. Clin Biomech 16:87101

Gajdosik RL, Vander Linden DW, McNair PJ, Riggin TJ, Albertson JS, Mattick DJ, Wegley JC (2004) Slow passive stretch and release characteristics of the calf muscles of older women with limited dorsiflexion range of motion. Clin Biomech (Bristol, Avon) 19:398-406

Gajdosik RL, Vander Linden DW, McNair PJ, Riggin TJ, Albertson JS, Mattick DJ, Wegley JC (2005) Viscoelastic properties of short calf muscle-tendon units of older women: effects of slow and fast passive dorsiflexion stretches in vivo. Eur J Appl Physiol 95:131-139

Hermens HJ, Freriks B, Disselhorst-Klug C, Rau G (2000) Development of recommendations for SEMG sensors and sensor placement procedures. J Electromyogr Kinesiol 10:361-374

Hufschmidt A, Mauritz KH (1985) Chronic transformation of muscle in spasticity: a peripheral contribution to increased tone. J Neurol Neurosurg Psychiatry 48:676-685

Lamontagne A, Malouin F, Richards CL, Dumas F (1997) Impaired viscoelastic behavior of spastic plantarflexors during passive stretch at different velocities. Clin Biomech 12:508-515

Magnusson SP (1998) Passive properties of human skeletal muscle during stretch maneuvers. A review. Scand J Med Sci Sports 8:65-77

Magnusson SP, Simonsen EB, Aagaard P, Gleim GW, McHugh MP, Kjaer M (1995) Viscoelastic response to repeated static stretching in the human hamstring muscle. Scand J Med Sci Sports $5: 342-347$
Magnusson SP, Simonsen EB, Aagaard P, Kjaer M (1996a) Biomechanical responses to repeated stretches in human hamstring muscle in vivo. Am J Sports Med 24:622-628

Magnusson SP, Simonsen EB, Aagaard P, Sorensen H, Kjaer M (1996b) A mechanism for altered flexibility in human skeletal muscle. J Physiol 497(Pt 1):291-298

Magnusson SP, Aagard P, Simonsen E, Bojsen-Moller F (1998) A biomechanical evaluation of cyclic and static stretch in human skeletal muscle. Int J Sports Med 19:310-316

Magnusson SP, Aagaard P, Larsson B, Kjaer M (2000a) Passive energy absorption by human muscle-tendon unit is unaffected by increase in intramuscular temperature. J Appl Physiol 88:12151220

Magnusson SP, Aagaard P, Nielson JJ (2000b) Passive energy return after repeated stretches of the hamstring muscle-tendon unit. Med Sci Sports Exerc 32:1160-1164

Mandel J (1969) Aperçu sur les principaux comportements rhélogiques. In: Persoz B (ed) La rhéololgie. Masson \& Cie, Paris, pp $1-17$

McFaull SR, Lamontagne A (1998) In vivo measurement of the passive viscoelastic properties of the human knee joint. Hum Mov Sci 17:139-165

McNair PJ, Portero P (2005) Using isokinetic dynamometers for measurements associated with tissue extensibility. Isokinet Exerc Sci 13:53-56

McNair PJ, Dombroski EW, Hewson DJ, Stanley SN (2001) Stretching at the ankle joint: viscoelastic responses to holds and continuous passive motion. Med Sci Sports Exerc 33:354358

McNair PJ, Hewson DJ, Dombroski E, Stanley SN (2002) Stiffness and passive peak force changes at the ankle joint: the effect of different joint angular velocities. Clin Biomech (Bristol, Avon) 17:536-540

Nordez A, Cornu C, McNair P (2006) Acute effects of static stretching on passive stiffness of the hamstring muscles calculated using different mathematical models. Clin Biomech 21:755-760

Nordez A, Casari P, Cornu C (2008a) Accuracy of Biodex system 3 pro isokinetic dynamometer in passive mode. Med Eng Phys

Nordez A, McNair PJ, Casari P, Cornu C (2008b) Acute changes in hamstrings musculo-articular dissipative properties induced by cyclic and static stretching. Int J Sports Med

Persoz B (1960) Introduction à l'étude de la rhéologie. Dunod, Paris, France

Rabita G, Dupont L, Thevenon A, Lensel-Corbeil G, Pérot C, Vanvelcenaher J (2005) Quantitative assessement of the velocity-dependent increase in resistance to passive stretch in spastic platarflexors. Clin Biomech 20:745-753

Reid DA, McNair PJ (2004) Passive force, angle, and stiffness changes after stretching of hamstring muscles. Med Sci Sports Exerc 36:1944-1948

Riemann BL, DeMont RG, Ryu K, Lephart SM (2001) The effects of sex, joint angle, and the gastrocnemius muscle on passive ankle joint complex stiffness. J Athl Train 36:369-375

Singer BJ, Dunne JW, Singer KP, Allison GT (2003) Velocity dependent passive platarflexor resistive torque in patients with acquired brain injury. Clin Biomech 18:157-165

Taylor CD, Dalton JD, Seaber AV, Garrett WE (1990) Viscoelastic properties of muscle-tendon units. The biomechanical effetcts of stretching. Am J Sports Med 18:300-309

Zatsiorski V (1998) Kinematics of human motion. Human Kinetics, Champaign 